PROCEEDINGS OF THE

AMERICAN MATHEMATICAL SOCIETY

Volume 130, Number 6, Pages 1833-1842

S 0002-9939(01)06224-4

Article electronically published on October 17, 2001

\title{
ON TRANSLATIONS OF SUBSETS OF THE REAL LINE
}

\author{
JACEK CICHOŃ, ANDRZEJ JASIŃSKI, ANASTASIS KAMBURELIS, \\ AND PRZEMYSŁAW SZCZEPANIAK
}

(Communicated by Alan Dow)

\begin{abstract}
In this paper we discuss various questions connected with translations of subsets of the real line. Most of these questions originate from W. Sierpiński. We discuss the number of translations a single subset of the reals may have. Later we discuss almost invariant subsets of Abelian groups.
\end{abstract}

\section{IntRoduCtion}

We shall use standard set theoretical notation. By $|X|$ we denote the cardinality of set $X$. If $\kappa$ is a cardinal number, then by $c f(\kappa)$ we denote its cofinality. We identify the first infinite cardinal number $\omega$ with the set of natural numbers. By c we denote the cardinality of continuum, i.e., $\mathbf{c}=2^{\omega}$. The Continuum Hypothesis (denoted by $\mathbf{C H}$ ) is the statement that $\mathbf{c}=\omega_{1}$. By MA we denote the Martin's Axiom (see [J]).

For any set $X$ and a cardinal number $\kappa$ let $[X]^{\kappa}$ (resp. $[X]^{<\kappa}$ ) be the family of all subsets of $X$ of cardinality $\kappa$ (resp. less than $\kappa$ ). By $A^{c}$ we denote the complement of the set $A$. The real line is denoted by $\mathbf{R}$. Let $\mathcal{L}$ be the $\sigma$-ideal of Lebesgue measure zero subsets of $\mathbf{R}$. Also let $\mathcal{K}$ be the $\sigma$-ideal of first category subsets of $\mathbf{R}$.

If $J$ is an ideal of subsets of a set $\mathrm{X}$ we define the following cardinal coefficients: $\operatorname{add}(J)=\min \{|S|: S \subseteq J$ and $\bigcup S \notin J\}, \operatorname{cov}(J)=\min \{|S|: S \subseteq J$ and $\bigcup S=$ $X\}$, and $\operatorname{non}(J)=\min \{|T|: T \subseteq X$ and $T \notin J\}$. If some cardinal number is not defined, then we assume that this number is $\infty$, where $\infty>\kappa$ for every cardinal number $\kappa$. We write $H \triangleleft G$ if $H$ is a subgroup of the group $G$.

We would like to thank the referee for many helpful comments.

\section{Preliminaries}

Suppose that $(G,+)$ is a group. For $A, B \subseteq G$ and $g \in G$ define $A \pm g=\{a \pm g$ : $a \in A\}$ and $A \pm B=\{a \pm b: a \in A \wedge b \in B\}$. Let $J$ be an ideal of subsets of $G$. We say that $J$ is invariant if $A+g \in J$ for every $A \in J$ and $g \in G$.

Convention. In what follows, we always assume that $G$ is an Abelian group and $J$ is an invariant ideal of subsets of $G$.

Received by the editors July 6, 2000 and, in revised form, December 8, 2000.

2000 Mathematics Subject Classification. Primary 03E15; Secondary 28A05.

Key words and phrases. Lebesgue measure, Baire property, almost invariant sets.

(C)2001 American Mathematical Society 
For $A \subseteq G$ define $\operatorname{Fix}(A, J)=\{g \in G:(A+g) \triangle A \in J\}$. Here $\triangle$ denotes the symmetric difference of sets. Then $\operatorname{Fix}(A, J)$ is a subgroup of $G$. Note also that $\operatorname{Fix}\left(A^{c}, J\right)=\operatorname{Fix}(A, J)$

Definition. We say that $A$ is $J$-almost invariant if $\operatorname{Fix}(A, J)=G$.

Lemma 2.1. Let $A \subseteq G$. The following conditions are equivalent:

1. A is $J$-almost invariant, i.e., $\forall g \in G(A+g) \triangle A \in J$;

2. $\forall g \in G(A+g) \backslash A \in J$;

3. $\forall g \in G \quad A \backslash(A+g) \in J$.

Note that the family of all $J$-almost invariant sets forms a field of subsets of $G$. This field is add( $J)$-complete, i.e., it is closed under taking unions of length less than $\operatorname{add}(J)$. If $A \in J$, then obviously $A$ and $A^{c}$ are $J$-almost invariant. We consider such sets as trivial $J$-almost invariant sets. Otherwise we say that $A$ is nontrivial.

Let us note the following easy fact concerning translations.

Lemma 2.2. If $G=A \cup B$ where $A \cap B=\emptyset$ and $T \subseteq G$, then either $T+g \subseteq B$ for some $g \in G$ or else $A-T=G$.

This gives us another simple characterization of $J$-almost invariant sets.

Lemma 2.3. $A$ is $J$-almost invariant iff $\forall T \subseteq A$ if $T \notin J$, then $A-T=G$.

We omit the easy proof. By this lemma $A-A=G$, if $A$ is $J$-almost invariant and $A \notin J$. This shows that certain almost invariant subsets of $\mathbf{R}$ are destroyed after forcing a new real.

The next observation gives a condition on $J$ ensuring that all $J$-almost invariant sets are trivial. This was also proved in $[\mathrm{L}]$ (Theorem 2).

Proposition 2.4. Suppose that $J$ satisfies the following condition:

If $A \notin J$, then there exists $T \subseteq A$ such that $T \notin J$ and $|T|<\operatorname{cov}(J)$.

Then all $J$-almost invariant sets are trivial.

Proof. Suppose on the contrary that $A$ is a nontrivial $J$-almost invariant set. Then so is $B=A^{c}$ and $A, B \notin J$. Since our assumptions are symmetric, we may assume that $B$ cannot be covered by less than $\operatorname{cov}(J)$ sets from $J$. Choose $T \subseteq A$ such that $T \notin J$ and $|T|<\operatorname{cov}(J)$. By Lemma 2.3 we have $A-T=G$. That is, $G=\bigcup_{g \in T} A-g$. So $B=\bigcup_{g \in T}(A-g) \cap B$. But $A$ is $J$-almost invariant, so $(A-g) \cap B \in J$ for every $g \in G$. We have just covered the set $B$ by less than $\operatorname{cov}(J)$ sets from $J$. A contradiction.

Corollary 2.5. Suppose $\omega \leq \kappa<|G|$. Let $G=A \cup B, A \cap B=\emptyset$ and $|A|=|B|=$ $|G|$. Then there exists $g \in G$ such that $|(A+g) \cap B| \geq \kappa$.

Proof. Let $J=\{X \subseteq G:|X|<\kappa\}$. Then $\operatorname{cov}(J)=|G|$ and $J$ satisfies the condition from Proposition 2.4. So we conclude that $A$ is not $J$-almost invariant, so there exists $g \in G$ such that $(A+g) \backslash A=(A+g) \cap B \notin J$.

\section{Translations of subsets of the Real line}

In this section we discuss some translation properties of subsets of the real line $\mathbf{R}$. Hence our basic group is the real line and the basic ideal is $\{\emptyset\}$. Sierpiński ( $\underline{\mathbf{S} 2}$ ) asked how many distinct translations a given set $A \subseteq \mathbf{R}$ has, i.e., what the 
cardinality of $\operatorname{Tr}(A):=\{A+g: g \in \mathbf{R}\}$ is. It is obvious that $|\operatorname{Tr}(A)|=1$ iff $A=\emptyset$ or $A=\mathbf{R}$. It is also easy to see that $|\operatorname{Tr}(A)|=\mathbf{c}$ for every bounded nonempty set $A \subseteq \mathbf{R}$. Recall that $\operatorname{Fix}(A)=\{g \in \mathbf{R}: A+g=A\}$ is a subgroup of $\mathbf{R}$. It is easy to observe that $|\operatorname{Tr}(A)|=|\mathbf{R} / \operatorname{Fix}(A)|$. But $\mathbf{R}$ is a divisible group. Therefore, if $|\operatorname{Tr}(A)| \geq 2$, then $|\operatorname{Tr}(A)| \geq \omega$.

For any cardinal number $\kappa$ such that $\omega \leq \kappa \leq \mathbf{c}$ one can find $G \triangleleft \mathbf{R}$ such that $|\operatorname{Tr}(G)|=\kappa$. To see this, let $\left\{x_{\alpha}: \alpha<\mathbf{c}\right\}$ be any Hamel basis and let $G=$ $\operatorname{LSP}\left(x_{\alpha}: \alpha \geq \kappa\right)$ be the linear space over rationals generated by the set $\left\{x_{\alpha}: \alpha \geq \kappa\right\}$ (note that $\operatorname{Fix}(G)=G$ for $G \triangleleft \mathbf{R}$ ).

A classical construction yields a Vitali set $V \subset(0,1)$. Then $|\operatorname{Tr}(V)|=\mathbf{c}$. But there exists a Vitali set with only $\omega$ translations. ${ }^{1}$ Just take a Hamel basis $H$ with $1 \in H$ and let $V=\operatorname{LSP}(H \backslash\{1\})$.

All the above-mentioned examples used "strange" subsets of $\mathbf{R}$. The following theorem was proved by Sierpiński (see [S2]) under the additional assumption of the Continuum Hypothesis.

Theorem 3.1. Suppose that $A \subseteq \mathbf{R}$ is Lebesgue measurable or has the Baire property. Then $|\operatorname{Tr}(A)| \in\{1, \mathbf{c}\}$.

Proof. Let us prove the theorem for the measure case. Let $G=\operatorname{Fix}(A)$. If $G$ is a discrete subgroup of the real line, then $|\mathbf{R} / G|=\mathbf{c}$. Hence we may assume that $G$ is a dense subgroup of the real line. If $A=\emptyset$ or $A=\mathbf{R}$, then $|\operatorname{Tr}(A)|=1$. Hence we may assume that $\emptyset \neq A \neq \mathbf{R}$. We claim that $A \in \mathcal{L}$ or that $A^{c} \in \mathcal{L}$. Suppose otherwise. Then, by Steinhaus' theorem (see $\overline{\mathrm{Ox}}$ ) the interior of the set $A-A^{c}$ is nonempty, so $\left(A-A^{c}\right) \cap G \neq \emptyset$. Let $a \in A, b \in A^{c}$ and $g \in G$ be such that $g=a-b$. Then $b=a-g$ and this is impossible, since $G=\operatorname{Fix}(A)$. Since $\operatorname{Fix}(A)=\operatorname{Fix}\left(A^{c}\right)$ we may assume that $A \in \mathcal{L}$. Let $a \in A$. Then $a+G \subseteq A$, so $G \in \mathcal{L}$. Let $H=\left\{(x, y) \in \mathbf{R}^{2}: x-y \in G\right\}$. Then $H$ is a Lebesgue measure zero subset of the plane $\mathbf{R}^{2}$. Hence, by Mycielski's theorem (see [M2]) there exists a perfect subset $P$ of the real line $\mathbf{R}$ such that $P \times P \backslash\{(x, x): x \in \mathbf{R}\} \subseteq \mathbf{R}^{2} \backslash H$. Hence, if $t_{1}, t_{2} \in P$ and $t_{1} \neq t_{2}$, then $\left(t_{1}, t_{2}\right) \notin H$, hence $t_{1}-t_{2} \notin G$, so $G+t_{1} \neq G+t_{2}$. Therefore $|\mathbf{R} / G|=\mathbf{c}$.

The proof of the second case of the theorem is similar to the one presented. Instead of Steinhaus' theorem for the Lebesgue measure it is necessary to use Steinhaus' theorem for the Baire property (see $[\mathrm{Ox}]$ ) and Mycielski's theorem for measure should be replaced by Mycielski's theorem for the Baire property (see [M1]).

Let us consider the Cantor dyadic group $\left(2^{\omega},+\right.$ ) (where $2=\{0,1\}$ and + is the usual pointwise addition modulo 2). Let us fix a natural number $n$ and let us consider the group $G_{n}=\left\{x \in 2^{\omega}: \forall i<n x(i)=0\right\}$. Then $\left|2^{\omega} / G_{n}\right|=2^{n}$. Hence there are sets $A \subseteq 2^{\omega}$ such that $1<|\operatorname{Tr}(A)|<\omega$.

Lemma 3.2. Let $A \subseteq 2^{\omega}$. Then $|\operatorname{Tr}(A)| \in\left\{2^{n}: n \in \omega\right\} \cup\{\kappa: \omega \leq \kappa \leq \mathbf{c}\}$. If $A$ is a Lebesgue measurable set or has the Baire property, then $|\operatorname{Tr}(A)| \in\left\{2^{n}: n \in\right.$ $\omega\} \cup\{\mathbf{c}\}$.

Proof. Let $G=\operatorname{Fix}(A)$. Suppose that $L=2^{\omega} / G$ is finite. Then $L$ is a linear space over the field $\mathbf{Z}_{2}$, hence $|L|=2^{n}$ for some natural number $n$. Notice that Steinhaus and Mycielski's theorems hold for Lebesgue measurable subsets of $2^{\omega}$ or sets with the Baire property. Hence we may repeat arguments from the previous theorem for

\footnotetext{
${ }^{1}$ This remark is due to J. Pawlikowski.
} 
the topological group $2^{\omega}$ and show that $|\operatorname{Tr}(A)| \leq \omega$ or $|\operatorname{Tr}(A)|=\mathbf{c}$ for Lebesgue measurable subsets $A \subseteq 2^{\omega}$ or sets with the Baire property.

\section{Almost invariant subsets of uncountable groups}

For an infinite group $G$ let $F_{G}=\{A \subseteq G:|A|<|G|\}$ be the Frechet ideal. Note that $F_{G}$ is invariant and $\operatorname{add}\left(F_{G}\right)=c f(|G|)$.

Definition. We say that $A \subseteq G$ is almost invariant if $A$ is $F_{G}$-almost invariant, i.e., if $|(A+g) \triangle A|<|G|$ for every $g \in G$.

In this section we consider almost invariant sets $A \subseteq G$ where $G$ is an uncountable (Abelian) group. We start our considerations with a technical lemma which extends the original Sierpiński's construction.

Lemma 4.1. Suppose that $G$ is an uncountable group. Let $|G|=\kappa$. Suppose that we are given $A \subseteq G$ and a sequence $\left\langle P_{\alpha}: \alpha<\kappa\right\rangle$ of subsets of $G$ such that

$$
\forall \alpha<\kappa \forall B \in[G]^{<\kappa}\left|\bigcap_{g \in B}(A-g) \cap P_{\alpha}\right|=\kappa .
$$

Let $\left\langle G_{\alpha}: \alpha<\kappa\right\rangle$ be an increasing family of subgroups of $G$ such that $\bigcup_{\alpha<\kappa} G_{\alpha}=G$ and $\left|G_{\alpha}\right|<\kappa$ for each $\alpha<\kappa$ (if not specified otherwise we let $G_{\alpha}$ be the subgroup of $G$ generated by $\left\langle g_{\beta}: \beta \leq \alpha\right\rangle$ where $\left\langle g_{\beta}: \beta<\kappa\right\rangle$ is some fixed enumeration of $\left.G\right)$.

Then there exists a sequence $\left\langle t_{\alpha}: \alpha<\kappa\right\rangle \subseteq G$ such that:

1. the sets $G_{\alpha}+t_{\alpha}$ are pairwise disjoint;

2. $G_{\alpha}+t_{\alpha} \subseteq A$ for $\alpha<\kappa$;

3. $\left(G_{\alpha}+t_{\alpha}\right) \cap P_{\alpha} \neq \emptyset$ for $\alpha<\kappa$;

4. if $Z \subseteq \kappa$ is cofinal in $\kappa$, then $\bigcup_{\alpha \in Z}\left(G_{\alpha}+t_{\alpha}\right)$ is an almost invariant set of cardinality $\kappa$.

Proof. First note that $(B+t) \cap C \neq \emptyset$ iff $t \in C-B$. Moreover $t \in \bigcap_{g \in B}(A-g)$ iff $B+t \subseteq A$. Build $\left\langle t_{\alpha}: \alpha<\kappa\right\rangle$ by induction. Suppose that $\beta<\kappa$ and $\left\langle t_{\alpha}: \alpha<\beta\right\rangle$ has been defined. Then, it is possible to choose $t_{\beta}$ such that

$$
t_{\beta} \in \bigcap_{g \in G_{\beta}}(A-g) \cap P_{\beta}
$$

and

$$
\left(G_{\beta}+t_{\beta}\right) \cap \bigcup_{\alpha<\beta}\left(G_{\alpha}+t_{\alpha}\right)=\emptyset
$$

(note that $\left|G_{\alpha}+t_{\alpha}\right|=\left|G_{\alpha}\right| \leq\left|G_{\beta}\right|<\kappa$ ). The sequence $\left\langle t_{\alpha}: \alpha<\kappa\right\rangle$ is the required one. Conditions 1,2 and 3 are satisfied. Let $Z \subseteq \kappa$ be cofinal in $\kappa$ and $S=\bigcup_{\alpha \in Z}\left(G_{\alpha}+t_{\alpha}\right)$. Fix $g \in G$ and let $\beta<\kappa$ be such that $g \in G_{\alpha}$ for every $\alpha \geq \beta$. Then $G_{\alpha}+t_{\alpha}+g=\left(G_{\alpha}+g\right)+t_{\alpha}=G_{\alpha}+t_{\alpha}$. Hence

$$
(S+g) \backslash S \subseteq \bigcup_{\alpha<\beta}\left(G_{\alpha}+g\right)
$$

and this last union has cardinality less than $\kappa$. In the end it suffices to observe that $|S|=|Z| \cdot \sup _{\alpha \in Z}\left|G_{\alpha}\right|=\kappa$.

It follows easily from Lemma 4.1 that nontrivial almost invariant sets exist in every uncountable group. The following observation extends Sierpiński's result from S1. 
Proposition 4.2. Suppose that $G$ is an uncountable group and $2 \leq \lambda \leq|G|$. Then there exists a decomposition of $G$ into $\lambda$ nontrivial almost invariant sets.

Proof. Let $\kappa=|G|$. We apply Lemma 4.1 (with $A=P_{\alpha}=G$ ) and obtain a sequence $\left\langle t_{\alpha}: \alpha<\kappa\right\rangle$. Write $\kappa=\bigcup_{\alpha<\kappa} Z_{\alpha}$ where $Z_{\alpha}$ 's are pairwise disjoint sets of cardinality $\kappa$. Put $S_{\alpha}=\bigcup_{\beta \in Z_{\alpha}}\left(G_{\beta}+t_{\beta}\right)$. It follows from Lemma 4.1 that $S_{\alpha}$ 's are pairwise disjoint almost invariant sets of cardinality $\kappa$.

Suppose first that $2 \leq \lambda<c f(\kappa)$. Then the set $\bigcup_{\alpha<\lambda} S_{\alpha}$ is almost invariant because $c f(\kappa)=\operatorname{add}\left(F_{G}\right)$. Therefore the remainder $R=G \backslash \bigcup_{\alpha<\lambda} S_{\alpha}$ is also almost invariant. Hence $\left\{S_{\alpha}: \alpha<\lambda\right\} \cup\{R\}$ is the required decomposition (if $\lambda$ is finite then just glue $R$ with $S_{0}$ ).

Suppose now that $\operatorname{cf}(\kappa) \leq \lambda \leq \kappa$. The remainder $R=G \backslash \bigcup_{\alpha<\lambda} S_{\alpha}$ has cardinality at most $\kappa$. Write $R=\bigcup_{\alpha<\lambda} R_{\alpha}$ where $R_{\alpha}$ 's are pairwise disjoint sets of cardinality less than $\kappa$ (some of them may be empty). Put $T_{\alpha}=S_{\alpha} \cup R_{\alpha}$. Clearly $\left\{T_{\alpha}: \alpha<\lambda\right\}$ works.

We can ask whether a given set $A \subseteq G$ with $|A|=|G|$ can be decomposed into many nontrivial almost invariant sets. Of course this is not always possible (note that a nontrivial almost invariant subset of $\mathbf{R}$ must be unbounded). But if $A$ itself is almost invariant, then we can repeat the construction from Lemma 4.1 inside $A$. It follows in particular that there are no almost invariant "atoms". In fact we can prove a bit more. Recall that two sets $A, B \subseteq G$ are almost disjoint if $|A \cap B|<|G|$.

Proposition 4.3. Suppose that $G$ is an uncountable group and $A$ is an almost invariant subset of $G$ such that $|A|=|G|$. Then $A$ contains cf $(|G|)^{+}$nontrivial almost invariant sets which are pairwise almost disjoint.

Proof. Let $\kappa=|G|$ and let $A$ be almost invariant and $|A|=\kappa$. Assume first that $\kappa$ is a regular cardinal. Then $\left|\bigcap_{g \in B}(A-g)\right|=\kappa$ for every $B \in[G]^{<\kappa}$. Let us apply Lemma 4.1 for the set $A$ (with $P_{\alpha}=G$ ). Let $\mathcal{F} \subseteq[\kappa]^{\kappa}$ be a family consisting of pairwise almost disjoint sets such that $|\mathcal{F}|=\kappa^{+}$. For each $X \in \mathcal{F}$ we put $X^{*}=\bigcup_{\alpha \in X}\left(G_{\alpha}+t_{\alpha}\right)$. Then $\left\{X^{*}: X \in \mathcal{F}\right\}$ is the required family.

Assume now that $\kappa$ is singular and $\lambda=c f(\kappa)$. Fix an increasing sequence of infinite cardinals $\left\langle\kappa_{\alpha}: \alpha<\lambda\right\rangle$ less than $\kappa$ and cofinal in $\kappa$. Also fix an increasing sequence $\left\langle H_{\alpha}: \alpha<\lambda\right\rangle$ of subgroups of $G$ such that $\left|H_{\alpha}\right| \leq \kappa_{\alpha}$ and $G=\bigcup_{\alpha<\lambda} H_{\alpha}$. We have to be more careful in the proof of the Lemma 4.1] Let $J_{\alpha}=\{B \subseteq G$ : $\left.|B| \leq \kappa_{\alpha}\right\}$. Then $J_{\alpha}$ is an invariant ideal. Put $G_{\alpha}=\operatorname{Fix}\left(A, J_{\alpha}\right) \cap H_{\alpha}$. Then $G_{\alpha} \triangleleft G$ and $G=\bigcup_{\alpha<\lambda} G_{\alpha}$. Now we can repeat the construction of the sequence $\left\langle t_{\alpha}: \alpha<\lambda\right\rangle$ exactly as in the proof of Lemma 4.1)(letting $P_{\alpha}=G$ ). To see this note that $\bigcap_{g \in G_{\alpha}}(A-g)$ has cardinality $\kappa$. Again, fix an almost disjoint family $\mathcal{F} \subseteq[\lambda]^{\lambda}$ such that $|\mathcal{F}|=\lambda^{+}$and for $X \in \mathcal{F}$ put $X^{*}=\bigcup_{\alpha \in X}\left(G_{\alpha}+t_{\alpha}\right)$. Then $\left\{X^{*}: X \in \mathcal{F}\right\}$ is as required.

Previous results imply that there are a lot of nontrivial almost invariant subsets of the real line. However, we shall show that none of them can be a Borel set.

Proposition 4.4. Suppose that $A, B$ are two disjoint Borel subsets of the real line such that $\mathbf{R}=A \cup B$ and $|A|=|B|=\mathbf{c}$. Then there exists a real number $x$ such that $|(A+x) \cap B|=\mathbf{c}$.

Proof. Suppose first that $\mathbf{C H}$ is false. Let us use Corollary [2.5 for $G=\mathbf{R}$ and $\kappa=\omega_{1}$. Let $x \in \mathbf{R}$ be such that $|(A+x) \cap B|>\omega$. But $(A+x) \cap B$ is a Borel set, hence $|(A+x) \cap B|=\mathbf{c}$. 
Suppose now that $\mathbf{C H}$ is true. Let us consider any generic extension $V^{\prime}$ of the universe $V$ in which $\mathbf{C H}$ is false. Then

$$
V^{\prime} \models(\exists x)(\exists P)(P \in \operatorname{Perf} \wedge(\forall t)(t \in P \rightarrow t \in(A+x) \cap B))
$$

where Perf denotes the space of all perfect subsets of the real line. Observe that this property of the universe $V^{\prime}$ is a $\Sigma_{2}^{1}$-sentence with parameters from the ground universe $V$, so by Shoenfield's absoluteness theorem (see [J]) it holds also in the universe $V$. Hence the theorem is proved.

With some additional assumptions we can extend the above proposition to the case when $A$ is an analytic set.

Proposition 4.5. Suppose that $A \subseteq \mathbf{R}$ is an uncountable analytic set such that its complement contains a perfect subset. Then $A$ is not almost invariant.

Proof. Let $B=A^{c}$. Both $A$ and $B$ have the Baire property. If $A, B \notin \mathcal{K}$, then we can find nonempty open intervals $I, J$ and a set $F \in \mathcal{K}$ such that $I \backslash F \subseteq A$ and $J \backslash F \subseteq B$. Let $x \in \mathbf{R}$ be such that $(I+x) \cap J \neq \emptyset$. Then $|(A+x) \cap B|=\mathbf{c}$ and hence $A$ is not almost invariant.

Therefore, we can assume that $A \in \mathcal{K}$ or $B \in \mathcal{K}$. For both sets there exists a canonical decomposition into $\omega_{1}$ Borel sets (constituents), which is absolute for $\omega_{1}$ preserving generic extensions. Say $A=\bigcup_{\alpha<\omega_{1}} A_{\alpha}$ and $B=\bigcup_{\beta<\omega_{1}} B_{\beta}$. If $\mathbf{C H}$ is false, then we add a single Cohen real $c$ to the universe $V$ and we work in $V[c]$. Assume that $B \in \mathcal{K}$ (the case when $A \in \mathcal{K}$ is similar). Note that if $x \in \mathbf{R} \cap V$, then the number $x+c$ is a Cohen real over $V$. Therefore $\mathbf{R} \cap V+c \subseteq A^{*}$, where by $A^{*}$ we denote the set $A$ encoded in $V[c]$. Hence $\mathbf{R} \cap V \subseteq A^{*}-c$ and so $B^{*} \cap V \subseteq\left(A^{*}-c\right) \cap B^{*}$. As $\left|B^{*} \cap V\right|=\mathbf{c}>\omega_{1}$ (by absoluteness $B^{*} \cap V$ is just the set $B$ in $V$ ) we can find $\alpha, \beta<\omega_{1}$ such that $\left|\left(A_{\alpha}^{*}-c\right) \cap B_{\beta}^{*}\right|=\mathbf{c}$. Thus $V[c]$ models the absolute sentence " $(\exists x)\left(\left(A_{\alpha}^{*}-x\right) \cap B_{\beta}^{*}\right.$ is uncountable $)$ " and we conclude that (in $V$ ) there exists $x$ such that $(A-x) \cap B$ is uncountable.

If $\mathbf{C H}$ is true we first extend the universe by adding $\omega_{2}$ Sacks reals either to the set $A$ (if $A \in \mathcal{K}$ ) or to the set $B$ (if $B \in \mathcal{K}$ ). In both cases it is possible because $A$ and $B$ contain perfect subsets. Then we can argue as we did previously.

Corollary 4.6. Assume that every co-analytic set $B \subseteq \mathbf{R}$ of cardinality $\mathbf{c}$ contains a perfect subset (this assumption follows e.g. from the negation of $\boldsymbol{C H}$ or from the existence of a measurable cardinal). Then all analytic almost invariant subsets of $\mathbf{R}$ are trivial.

Note that the proof of Proposition 4.5 breaks down when $B$ is a co-analytic set such that all its constituents are countable. Then it is impossible to force new elements to $B$ without collapsing $\omega_{1}$.

The next theorem, due to A. Miller 2 shows that such sets are possible in the constructible universe $L$.

Theorem 4.7 (A. Miller). If $V=L$, then there exists a nontrivial almost invariant $\Pi_{1}^{1}$ subset of $\mathbf{R}$. Similarly, there is such a subset of $2^{\omega}$.

Proof. To see that a given transfinite construction of a set of reals can be converted into the construction of a $\Pi_{1}^{1}$ set under $V=L$, what must be seen is that the construction itself can be coded into each real which is appearing at a given stage.

${ }^{2}$ We would like to thank Arnold Miller for his kind permission to include his result. 
In the transfinite construction of the sets $\left\langle t_{\alpha}+G_{\alpha}: \alpha<\omega_{1}\right\rangle$ it is enough to see that each $u \in t_{\alpha}+G_{\alpha}$ can code up an arbitrary real $z \in 2^{\omega}$. (In the proof below $z$ will be recursive in each $u \in t_{\alpha}+G_{\alpha}$, but more generally it would be enough for $z$ to be $\Delta_{1}^{1}$ in each $u$.) The real $z$ in turn can code up the construction of $\left\langle t_{\beta}+G_{\beta}: \beta<\alpha\right\rangle$ as well as the initial part of the $L$ hierarchy, i.e., some $L_{\gamma}$, in which it appears. This makes every real entering the set "self-constructible" and hence we can make our set $\bigcup_{\beta<\omega_{1}} t_{\beta}+G_{\beta}$ into a $\Pi_{1}^{1}$ set. (We suppose we can guarantee it is nontrivial by taking only the even $\beta$ 's.) If we also take $z$ not to be recursive in any $u \in \bigcup_{\beta<\alpha} t_{\beta}+G_{\beta}$, then this also automatically guarantees that $t_{\alpha}+G_{\alpha}$ is disjoint from $\bigcup_{\beta<\alpha} t_{\beta}+G_{\beta}$.

The coding argument is easier for the Cantor group $\left(2^{\omega},+\right)$ so we do it first.

Lemma 4.8. Given $G \subseteq 2^{\omega}$ countable and $z \in 2^{\omega}$ there exists $t \in 2^{\omega}$ such that $z \leq_{T} t+g$ for each $g \in G$. The proof shows that such $t$ can be found recursive in $z$ and any enumeration of $G$.

Proof. Let $G=\left\{g_{n}: n \in \omega\right\}$ be any enumeration. Let $\left\langle A_{n}: n \in \omega\right\rangle$ be a recursive partition of $\omega$ into infinite sets and for each $n$ let $A_{n}=\left\{k_{0}^{n}<k_{1}^{n}<k_{2}^{n}<\ldots\right\}$.

Define $t \in 2^{\omega}$ by

$$
t\left(k_{m}^{n}\right)+g_{n}\left(k_{m}^{n}\right)=z(m) .
$$

Then we have $z \leq_{T} t+g_{n}$ as required.

Lemma 4.9. Given $G \subseteq \mathbf{R}$ countable and $z \in 2^{\omega}$ there exists $t \in \mathbf{R}$ such that $z \leq_{T} t+g$ for each $g \in G$. The proof shows that such $t$ can be found recursive in $z$ and any enumeration of $G$.

Proof. Let $\left\{g_{n}: n \in \omega\right\}$ and $\left\langle A_{n}: n \in \omega\right\rangle$ be as in the proof above. Define $\epsilon_{n}=6^{-n}$ for $n \in \omega$ and let $J_{m}^{n}$ be the closed intervals of length $\epsilon_{n}$ defined by

$$
J_{m}^{n}=\left[m \epsilon_{n},(m+1) \epsilon_{n}\right] \text { for } m \in \mathbf{Z} .
$$

For each $n$ these intervals cover $\mathbf{R}$ and overlap only on their endpoints.

We will construct a sequence $\left\langle I_{n}: n \in \omega\right\rangle$ of closed intervals such that $\left|I_{n}\right| \geq \frac{1}{2} \epsilon_{n}$. The $t$ we want will be in the intersection of the $I_{n}$ 's.

Let $I_{0}=\mathbf{R}$. Suppose that $n+1=k_{i}^{j} \in A_{j}$. Given $I_{n}$ with $\left|I_{n}\right| \geq \frac{1}{2} \epsilon_{n}$, since $\epsilon_{n+1}=\frac{1}{6} \epsilon_{n}$ we know that $\left|I_{n}\right| \geq 3 \epsilon_{n+1}$ and therefore $g_{j}+I_{n}$ covers at least two consecutive intervals of length $\epsilon_{n+1}$, say

$$
J_{n+1}^{m} \cup J_{n+1}^{m+1} \subseteq g_{j}+I_{n} .
$$

Now choose $\hat{m} \in\{m, m+1\}$ so that $\hat{m}$ is even iff $z(i)=0$. Next choose $I_{n+1} \subseteq I_{n}$ with length at least $\frac{1}{2} \epsilon_{n+1}$ and so that

$$
g_{j}+I_{n+1} \subseteq \operatorname{interior}\left(J_{n+1}^{\hat{m}}\right) .
$$

This finishes the construction of the sequence $\left\langle I_{n}: n \in \omega\right\rangle$.

Let $t$ be the unique real in the intersection of all the $I_{n}$. We claim that $z \leq_{T} g_{j}+t$ for each $j$. To calculate $z(i)$ let $n+1=k_{i}^{j}$. By our construction there exists a unique $m$ such that $g_{j}+t \in J_{n+1}^{m}$ (we are assuming that our coding of real numbers is such that we can effectively find this $m)$. Then $z(i)=0$ iff $m$ is even.

Theorem 4.7 follows from these two lemmas using an argument similar to that of [M] (Lemma 7.22 and Theorem 7.21). 
Let us go back to Proposition 4.4 (Borel set case). We shall finish this section with a few comments. It is not true in general that given two perfect sets $P$ and $Q$ one can find $x \in \mathbf{R}$ such that $(P+x) \cap Q$ is uncountable. To see this just take two disjoint perfect sets $P$ and $Q$ which are subsets of the perfect set of algebraically independent reals. Then $|(P+x) \cap Q| \leq 1$ for every $x \in \mathbf{R}$.

The trick with a Cohen real from Proposition 4.5 gives the following alternative proof of Proposition 4.4 We can assume that $B \in \mathcal{K}$. Assume that $(A-x) \cap B$ is countable for every $x \in \mathbf{R}$. From Lusin-Novikov Uniformization Theorem (see $[\mathrm{K}])$ there exists a sequence of Borel measurable functions $f_{n}: \mathbf{R} \rightarrow \mathbf{R}$ such that $(A-x) \cap B \subseteq\left\{f_{n}(x): n<\omega\right\}$. For $y \in B$ we have $A-y \subseteq \bigcup_{n} f_{n}^{-1}(y)$. So there exists $n<\omega$ such that $f_{n}^{-1}(y) \notin \mathcal{K}$ for uncountably many $y \in B$. But this is impossible because the Boolean algebra $\operatorname{Borel}(\mathbf{R}) / \mathcal{K}$ satisfies the countable chain condition.

\section{Almost invariant subsets of COUntable Groups}

Here we assume that $G$ is an Abelian group and $|G|=\omega$. We shall write FIN for the Frechet ideal $F_{G}$.

It is easy to see that Lemma 4.1 generalizes to the case when $G$ can be written as a chain of finite subgroups (this simply means that every element of $G$ has finite order). This works e.g. for $\mathbf{C}_{p^{\infty}}$ or for the group of rational rotations of the unit circle.

Now consider $(\mathbf{Z},+)$. It is easy to see that almost invariant subsets of $\mathbf{Z}$ are of four types. They are equal (modulo FIN) to either $\emptyset$ or $\mathbf{Z}$ or $P:=\{n: n>0\}$ or $N:=\{n: n<0\}$. Thus $P$ and $N$ are nontrivial. But the next lemma shows that Lemma 4.1 cannot be generalized to all countable groups.

Lemma 5.1. Almost invariant subsets of $(\mathbf{Z} \times \mathbf{Z},+)$ are trivial. Similarly, almost invariant subsets of $(\mathbf{Q},+)$ are trivial.

Proof. Let $A \subseteq \mathbf{Z} \times \mathbf{Z}$. Suppose on the contrary that $A$ and $B:=A^{c}$ are almost invariant and $|A|=|B|=\omega$. Consider (horizontal) sections $A^{m}=\{n:\langle n, m\rangle \in A\}$. Each such section must be almost invariant in $\mathbf{Z}$ and therefore equal (modulo FIN) to $\emptyset, \mathbf{Z}, P$ or $N$. But observe that all sections must be of the same type. Thus (by passing to the complement if necessary) we may assume that all nonempty sections have the greatest element. Moreover, infinitely many sections must be nonempty. To obtain a contradiction it suffices to shift $A$ by $\langle 1,0\rangle$.

The case of $(\mathbf{Q},+)$ is left as an exercise.

\section{Almost invariant Sets modulo an ideal}

In this section we consider $J$-almost invariant subsets of the real line $\mathbf{R}$, where $J$ is an ideal of subsets of $\mathbf{R}$ with a Borel base. Recall that an ideal $J \subseteq \mathcal{P}(\mathbf{R})$ has a Borel base, if for every $X \in J$ there exists a Borel set $B \in J$ such that $X \subseteq B$. Well-known examples of such ideals are the $\sigma$-ideals $\mathcal{L}$ and $\mathcal{K}$. Recall also that a set $X \subseteq \mathbf{R}$ is called a Bernstein set if $X \cap P \neq \emptyset \neq X^{c} \cap P$ for every perfect set $P$.

Theorem 6.1. Suppose that $J$ is a proper invariant ideal on $\mathbf{R}$ with a Borel base such that $\operatorname{non}(J)=\mathbf{c}$. Then there exists a nontrivial $J$-almost invariant set which is also a Bernstein set.

Proof. Let $Z \subseteq \mathbf{c}$ be such that $|Z|=\left|Z^{c}\right|=\mathbf{c}$ and let $\left\langle P_{\alpha}: \alpha<\mathbf{c}\right\rangle$ be an enumeration of all perfect subsets of $\mathbf{R}$ such that each perfect set has an index 
both in $Z$ and $Z^{c}$. We use Lemma 4.1 (with $A=\mathbf{R}$ ) and obtain a sequence $\left\langle t_{\alpha}: \alpha<\mathbf{c}\right\rangle$. Put $X=\bigcup_{\alpha \in Z}\left(G_{\alpha}+t_{\alpha}\right)$. It is easy to see that $X$ is a Bernstein set. From Lemma 4.1 we know that $X$ is almost invariant. Therefore $X$ is $J$-almost invariant because $\operatorname{non}(J)=\mathbf{c}$. It suffices to show that $X \notin J$ and $X^{c} \notin J$. It follows from the fact that $X$ (and also $X^{c}$ ) is a Bernstein set. Assume e.g. that $X \in J$. Then there exists a Borel set $B \in J$ such that $X \subseteq B$. Its complement $B^{c}$ is an uncountable Borel set and therefore it contains a perfect set $P \subseteq B^{c}$. But $X \cap P \neq \emptyset$. A contradiction.

It is a well-known fact that Martin's Axiom implies that $\operatorname{add}(\mathcal{L})=\operatorname{non}(\mathcal{L})=\mathbf{c}$ and also $\operatorname{add}(\mathcal{K})=\operatorname{non}(\mathcal{K})=\mathbf{c}($ see $[\mathrm{J}])$. Thus we have

Corollary 6.2. Martin's Axiom implies that there are nontrivial $\mathcal{L}$-almost invariant sets and nontrivial $\mathcal{K}$-almost invariant sets. In fact under $\boldsymbol{M A}$ there is a set which is simultaneously nontrivial $\mathcal{L}$-almost invariant and nontrivial $\mathcal{K}$-almost invariant.

$\mathrm{W} \AA^{3}$ show that this cannot be proved in the theory $\mathbf{Z F C}$ alone.

Theorem 6.3. Let $M$ be a transitive model of $\boldsymbol{Z} \boldsymbol{F} \boldsymbol{C}+\boldsymbol{C H}$. Add $\omega_{2}$ random reals to $M$ (by measure algebra). Then in the generic extension $M\left[r_{\alpha}: \alpha<\omega_{2}\right]$ there exists a nontrivial $\mathcal{K}$-almost invariant set but there are no nontrivial $\mathcal{L}$-almost invariant sets.

Proof. Let $N=M\left[r_{\alpha}: \alpha<\omega_{2}\right]$. It is well known that in the model $N$ we have $\operatorname{cov}(\mathcal{L})=\omega_{2}=\mathbf{c}$. By Rothberger's theorem also $\operatorname{non}(\mathcal{K})=\mathbf{c}$ in $N$. Hence by Theorem 6.1 there exists a nontrivial $\mathcal{K}$-almost invariant set.

To show that there are no nontrivial $\mathcal{L}$-almost invariant sets we shall use Proposition 2.4 for the $\sigma$-ideal $\mathcal{L}$. We have to show that in $N$ the following is true:

$$
\text { if } X=\left\{x_{\alpha}: \alpha<\omega_{2}\right\} \notin \mathcal{L} \text {, then }\left\{x_{\alpha}: \alpha<\beta\right\} \notin \mathcal{L} \text { for some } \beta<\omega_{2} .
$$

This fact is probably less known but it is also a part of folklore (see [LM], Lemma 8). Let us sketch the main idea of the proof. We slightly abuse the notation by treating an object from $N$ as a name for it. The required $\beta$ will be the limit of an increasing sequence of ordinals $\left\{\beta_{\delta}: \delta<\omega_{1}\right\}$. Put $\beta_{0}=\omega$ and use limits at limit steps. Having $\beta_{\delta}$ look at the set $\mathcal{B}_{\delta}$ of all Borel sets from $\mathcal{L}$ coded in the submodel $M\left[r_{\alpha}: \alpha<\beta_{\delta}\right]$. Then $\left|\mathcal{B}_{\delta}\right|=\omega_{1}$ because this submodel satisfies $\mathbf{C H}$. As $X \notin \mathcal{L}$ it is possible to find $\beta_{\delta+1}>\beta_{\delta}$ such that for all $B \in \mathcal{B}_{\delta}$ there exists $\alpha<\beta_{\delta+1}$ such that $x_{\alpha} \notin B$ and $x_{\alpha} \in M\left[r_{\alpha}: \alpha<\beta_{\delta+1}\right]$. Put $Y=\left\{x_{\alpha}: \alpha<\beta\right\}$ and $M^{\prime}=M\left[r_{\alpha}: \alpha<\beta\right]$. It follows that $Y \in M^{\prime}$ and $M^{\prime} \mid=Y \notin \mathcal{L}$. But the complementary extension, from $M^{\prime}$ to $N$, is also by a measure algebra and it is known that it preserves the Lebesgue outer measure. It follows that $N \models Y \notin \mathcal{L}$.

The above proof can be dualized for the Baire category.

Theorem 6.4. Let $M$ be a transitive model of $\boldsymbol{Z F C}+\boldsymbol{C H}$. Add $\omega_{2}$ Cohen reals to $M$ (by product forcing). Then in the generic extension $M\left[c_{\alpha}: \alpha<\omega_{2}\right]$ there exists a nontrivial $\mathcal{L}$-almost invariant set but there are no nontrivial $\mathcal{K}$-almost invariant sets.

We don't know any model where there are no nontrivial $\mathcal{L}$-almost invariant sets or nontrivial $\mathcal{K}$-almost invariant sets. Surprisingly, in the iterated $\omega_{2}$ Sacks model we

\footnotetext{
3 This was also proved in $\underline{\mathrm{L}}$ (see Theorem 8).
} 
have the same effect as in Corollary 6.2; there exist:4 a set which is simultaneously nontrivial $\mathcal{L}$-almost invariant and nontrivial $\mathcal{K}$-almost invariant. Namely, it follows from the Covering Property Axiom (CPA; see $[\mathbf{C P}]$ ) that there exists a Hamel basis which consists of $\omega_{1}$ closed sets. It is possible then to repeat Sierpiński's construction.

\section{REFERENCES}

[CP] K. Ciesielski and J. Pawlikowski, A combinatorial core of the iterated perfect set model, preprint.

[J] T. Jech, Set Theory, Academic Press, New York, 1978. MR 80a:03062

[K] A. S. Kechris, Classical Descriptive Set Theory, Grad. Texts in Math. 156, Springer, 1995. MR 96e:03057

[L] M. Laczkovich, Two constructions of Sierpiński and some cardinal invariants of ideals, Real Analysis Exchange 24 (1998/9), 663-676. MR 2000f:03148

[LM] M. Laczkovich and A. W. Miller, Measurability of functions with approximately continuous vertical sections and measurable horizontal sections, Colloq. Math. 69 (1995), 299-308. MR 96k:28005

[M] A. W. Miller, Infinite combinatorics and definability, Ann. Pure Appl. Logic 41 (1989), 179-203. MR 90b:03070

[M1] J. Mycielski, Independent sets in topological algebras, Fund. Math. 55 (1964), 139-147. MR 30:3855

[M2] J. Mycielski, Algebraic independence and measure, Fund. Math. 61 (1967), 165-169. MR 37:361

[Ox] J. C. Oxtoby, Measure and category, Springer, Berlin, 1971. MR 52:14213

[S1] W. Sierpiński, Sur les translation des ensembles lineares, Fund. Math. 19 (1932), 22-28.

[S2] W. Sierpiński, Sur les translation des ensembles lineares, Fund. Math. 35 (1948), 159-164. MR 10:287d

Institute of Mathematics, Wroceaw University, Pl. Grunwaldzki 2/4, 50-384 WroCeAw, Poland

Institute of Mathematics, Wroceaw University, Pl. Grunwaldzki 2/4, 50-384 WroCŁaW, Poland

Institute of Mathematics, WrocŁaw University, Pl. Grunwaldzki 2/4, 50-384 WroCEAW, Poland

E-mail address: akamb@math.uni.wroc.pl

Institute of Mathematics, WrocŁaw University, Pl. Grunwaldzki 2/4, 50-384 WroCeaw, Poland

\footnotetext{
${ }^{4}$ This fact was communicated to us by K. Ciesielski and J. Pawlikowski.
} 